\title{
Optical-electronic complex for investigation of the processes of heat and mass transfer by laser contactless method
}

\author{
A.V. Vedyashkina1, B.S. Rinkevichyus ${ }^{2}$, I.N. Pavlov³, I.L. Raskovskaya4, A.V. Tolkachev5 \\ National Research University "MPEI" \\ 1 ORCID: 0ooo-0002-2670-7712, an.vedyashkina@gmail.com \\ 2 ORCID: 0000-0001-7336-1315, rinkevbs@mail.ru \\ 3 ORCID: 0000-0002-9014-6294, inpavlov@bk.ru \\ 4 ORCID: 0ooo-0002-1231-9479, raskovskail@mail.ru \\ 5 ORCID: 000o-0003-4185-892X, avtolk2008@yandex.ru
}

\begin{abstract}
The work is devoted to development and creation of an optoelectronic complex for determination of the inhomogeneous condensed media parameters, principle of which is based on the method of structured laser radiation caustics. This method is described for determining the parameters of diffusion layer of liquid according to the position of caustic surfaces during longitudinal probing by a plane laser beam. Special software is presented that allows determining the distribution of the refractive index in the diffusion layer of liquid by comparing theoretically calculated and experimental refractograms. The results of the complex operation for the diffusion layer arising between the electrolyte solution and water for different concentrations of the electrolyte solution are shown. New method for determining the temperature of the surface of a cold body placed in a transparent liquid is presented, it is based on determining the location of the singular points of caustic surfaces arising when the boundary layer is probed by the horizontal element of structured beam.
\end{abstract}

Keywords: diffusion layer of liquid, refraction, caustic, laser refractography.

\section{Introduction}

For non-disturbing noncontact diagnostics of heat and mass transfer processes, optical refraction methods are well recommended. There are some advantages, first of all optical measurements do not distort the heterogeneity under investigation, as in most cases the energy absorbed by the medium is rather small. Laser methods are low-inertia, which allows to perform accurate measurements of fast processes. Laser refraction methods allow us to investigate the field of the refractive index, which is then converted by indirect measurements into the sought field of another physical quantity [1].

Based on the use of lasers, new methods were developed to diagnose liquid and gas flows, namely, the method of laser Doppler anemometry, anemometry using particle images, photoluminescent methods, and a number of others [2]. At present, instruments using such methods are widely used in aero-hydrodynamic and thermophysical experiments.

In $[3,4]$, it was noted that for the diagnosis of stratified media under conditions of substantial refraction of optical radiation, it is advisable to use the method of laser refractography. The method is based on recording the refractive displacement of structured laser radiation (SLR) which probes optical inhomogeneity. In this case, the SLR is used, which is formed using special optical elements directly at the output of the radiation source [5]. This method of forming SLRs makes it possible to maintain its high coherence and to ensure a small divergence of the beams, which makes it possible to apply the concepts of geometric optics to describe SLIs. Structured beams are visualized in a section as a family of geometric figures, such 
as circles, line segments, or as a matrix of points. The distortion of elements of structured beams resulting from refraction is an informative parameter for solving the inverse problem.

Longitudinal probing of stratifications during measurements due to natural limitations in a full-scale experiment is used less frequently than traditional normal or oblique sounding, therefore caustics that occur in latter cases are mainly described in the literature. Nevertheless, when studying and monitoring thermophysical, hydrodynamic and chemical processes in laboratory conditions at the interfaces between media, there are often conditions for the emergence of stratifications of various nature [6,7], accompanied by significant gradients of the refractive index. In this case, the longitudinal sounding has proved to be very informative and it allows for the restoration of environmental parameters, including the cases, when caustics are produced and their geometric characteristics are later utilized.

The study of stratified media as well as transition (diffusion) layers is one of the important tasks of modern hydrophysics and oceanography. Stable density stratification is typical for natural reservoirs, including the World Ocean [8]. Often it is caused by the non-uniform heating of the water column, as well as the distribution of salinity in depth. The study of hydrophysical flows is mainly based on laboratory modeling and is associated with the creation of density stratification, measurement and control of its characteristics. Saline stratification is usually used in model laboratory experiments [9]. Through the vertical concentration reduction, scientists achieve the required density distribution. However, it is possible to create and maintain sufficiently long-term temperature stratification on unique installations [10].

In the following, under the stratified medium we will generally mean a two-dimensional inhomogeneous medium in which the component of the refractive index gradient in one of the coordinates significantly exceeds the component in the other coordinates. During SLR refraction in a stratified medium caustics can be observed both inside and at the exit of the medium where the rays are already straight lines [11].

In $[12,13]$, the possibility of applying the caustic method of laser radiation to determine the parameters of stratified media was described. In this approach, when solving the inverse problem of reconstructing the properties of optical inhomogeneities, the informative parameters are the coordinates of the caustic surfaces resulting from refraction.

At this stage of development of science and technology, the caustic method is widely used to diagnose stresses and deformations and to stop cracks in solids [14-16].

\section{Parameters determination of the diffusion layer of a liquid}

For diagnostics of the diffusion layer of a liquid, various types of SLR can be used; computer and experimental $3 \mathrm{D}$ visualization of the propagation of a cylindrical laser beam in the diffusion layer and caustics formed in this case are shown in [17].

In order to observe and register caustics arising during the propagation of the plane laser beam in the diffusion layer of liquid, optoelectronic complex was developed and created, the scheme and appearance of which are shown in Fig. 1a and 1b, respectively. 

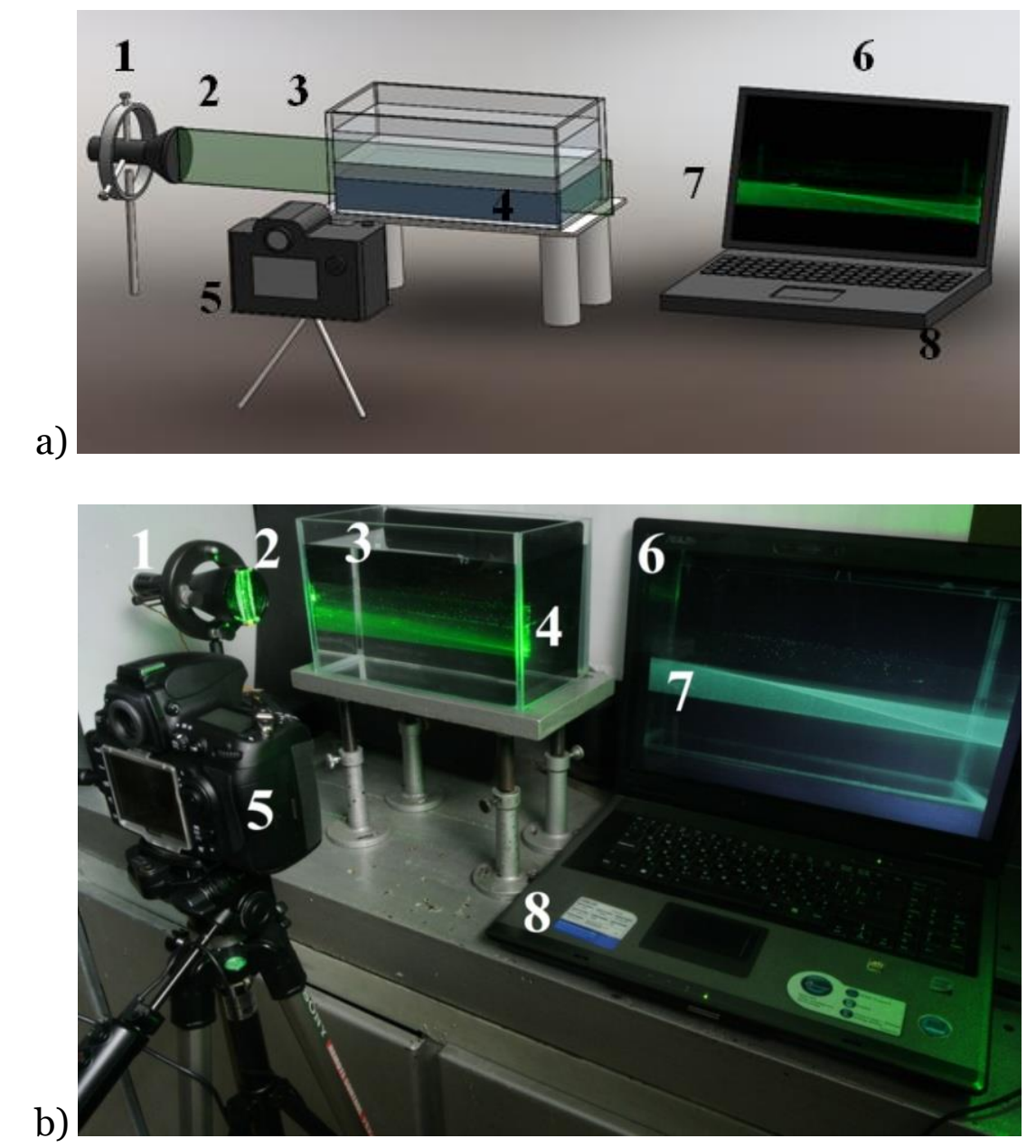

Fig. 1. Optical-electronic complex for registration of caustics of a plane laser beam in the diffusion layer of liquid ( $\mathrm{a}-$ scheme, $\mathrm{b}$ - appearance)

1 - laser, 2 - optical system, 3 - cuvette, 4 - diffusion layer,

5 - digital camera, 6 - PC, 7 - 2D refractogram, 8 - software

Compact laser module 1 with a wavelength $\lambda=532 \mathrm{~nm}$ is used as a radiation source. At the output of the laser, the optical system for forming structured laser radiation 2 is installed. The system consists of two cylindrical lenses, the relative position of which is chosen so that a collimated flat laser beam with a width of $48 \mathrm{~mm}$ is obtained at the output. The beam is directed to the cuvette parallel to the diffusion layer of liquid 4. The diffusion layer is located in the glass cuvette 3 . The laser beam refraction is registered using the digital camera 5 . This allows to achieve a quantitative estimate of the laser beam refraction after processing the resulting refractogram 7 using the developed software 8 on a personal computer 6 . The developed special software allows the simulation of the propagation of a plane laser beam in a diffusion layer of liquid, visualization of caustics arising from the refraction of the laser beam inside the inhomogeneous medium, and combination of them with the experimentally obtained refraction patterns, thereby determining the thickness of the diffusion layer of liquid in real time. The program also allows to make similar measurements of the thickness of the diffusion layer at different points in time during the experiment and to build the dependence of the thickness of the diffusion layer on time, followed by interpolation.

To create a diffusion layer, water and $\mathrm{KOH}$ electrolyte solution of various concentrations were used (table 1); the indicator distribution was described using the parametric model (1) [18]: 
Table 1 - Refractive indices for different concentrations of electrolyte $\mathrm{KOH}$

\begin{tabular}{|c|c|}
\hline Concentrations & Refractive indices \\
\hline $2 \mathrm{~mol} / \mathrm{l}$ & 1.3511 \\
\hline $4 \mathrm{~mol} / \mathrm{l}$ & 1.3670 \\
\hline $5 \mathrm{~mol} / \mathrm{l}$ & 1.3735 \\
\hline $6 \mathrm{~mol} / \mathrm{l}$ & 1.3800 \\
\hline $7 \mathrm{~mol} / \mathrm{l}$ & 1.3857 \\
\hline
\end{tabular}

$$
n(x)=\frac{n_{1}+n_{2}}{2}-\frac{n_{1}-n_{2}}{2} \text { th }\left(\frac{x-x_{s}}{h}\right),
$$

where $h$ is the characteristic half-width of the layer, $x_{s}$ is the middle of the layer.

The dynamics of experimental refractograms for the diffusion layer arising between an electrolyte solution with a concentration of $7 \mathrm{~mol} / \mathrm{l}$ and water obtained during the experiment at different points in time from the beginning of the layer creation are shown in Fig. 2

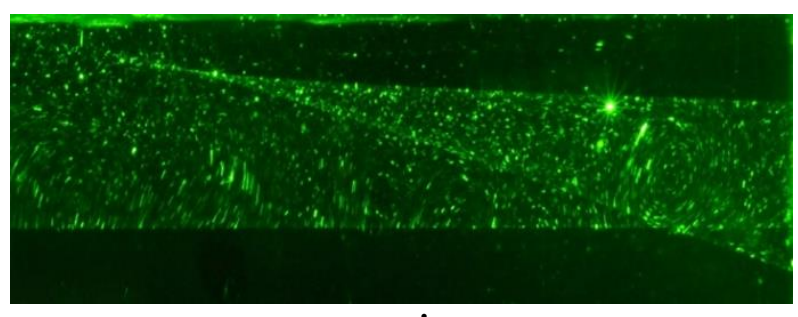

$2 \mathrm{~min}$

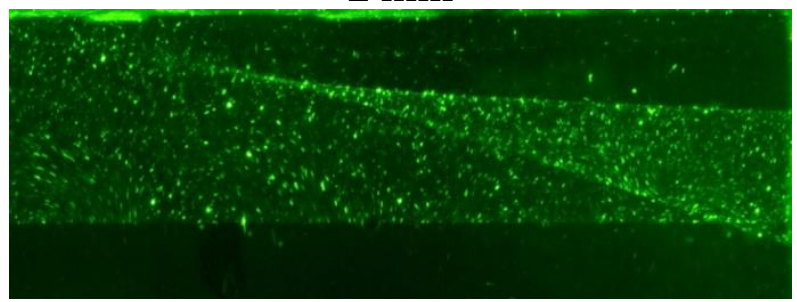

$6 \mathrm{~min}$

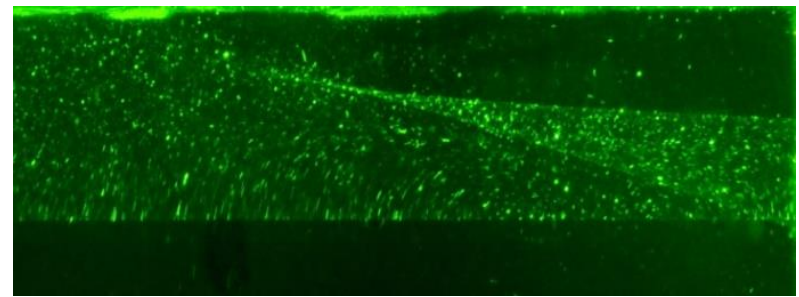

$10 \mathrm{~min}$

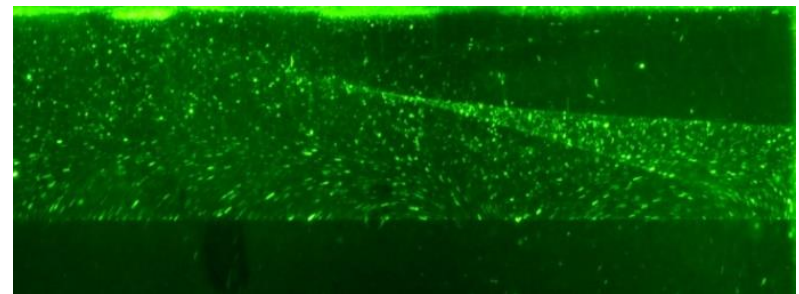

$14 \mathrm{~min}$

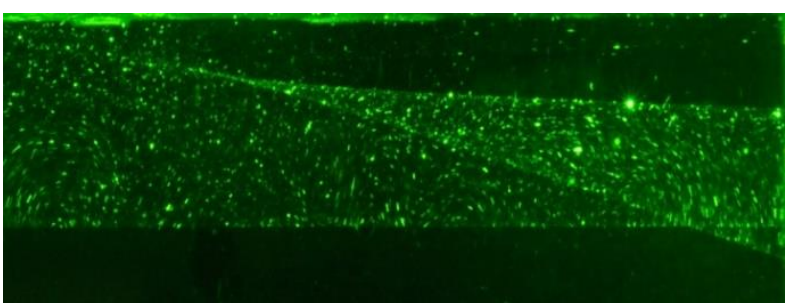

\section{$4 \mathrm{~min}$}

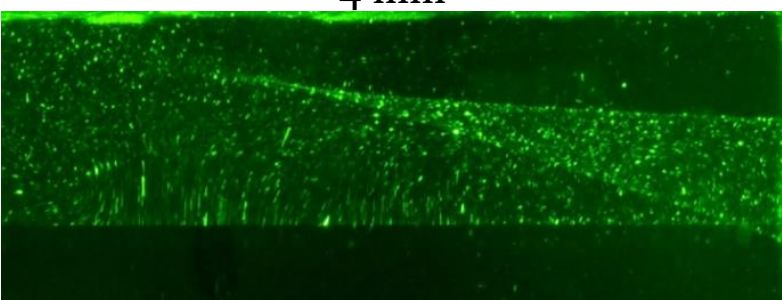

$8 \mathrm{~min}$

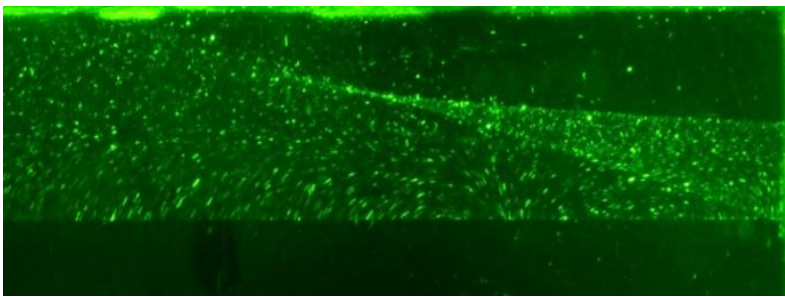

$12 \mathrm{~min}$

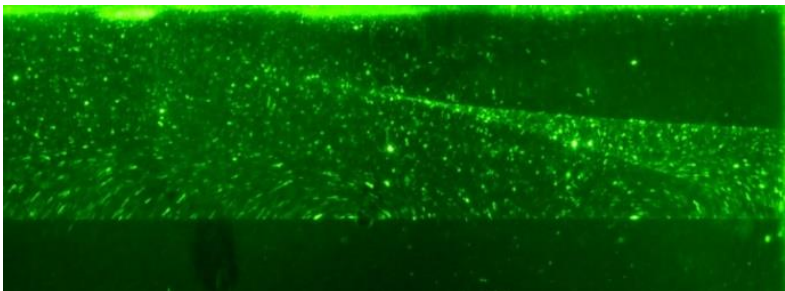

$16 \mathrm{~min}$

Fig. 2. Experimental 2D refractograms of the plane laser beam in the diffusion layer of liquid at various time points $(\Delta t=2 \mathrm{~min})$ 
Figure 3 shows the experimental graphs of the layer thickness $d$ in time for various $\Delta n=n_{1}-$ $n_{2}$.

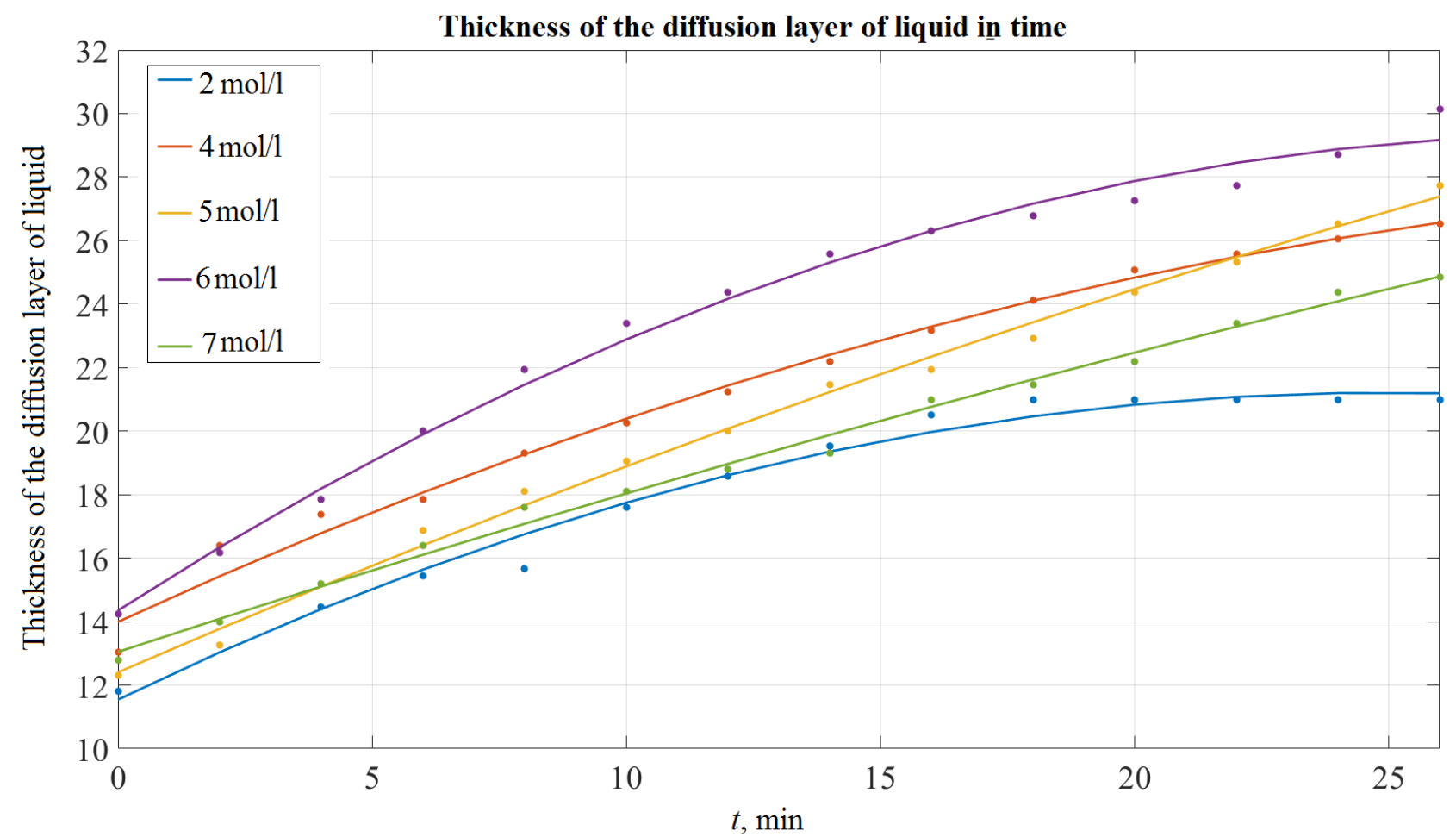

Fig. 3. Dependence between the thickness of the diffusion layer of liquid and time for different values of the electrolyte $\mathrm{KOH}$ concentration

Thus, the registration of refractograms at different times with equal intervals allows to estimate the dependence of the layer thickness on time.

Dependency graphs of the distribution of the refractive index and the gradient of the refractive index are shown in Fig. 4. 

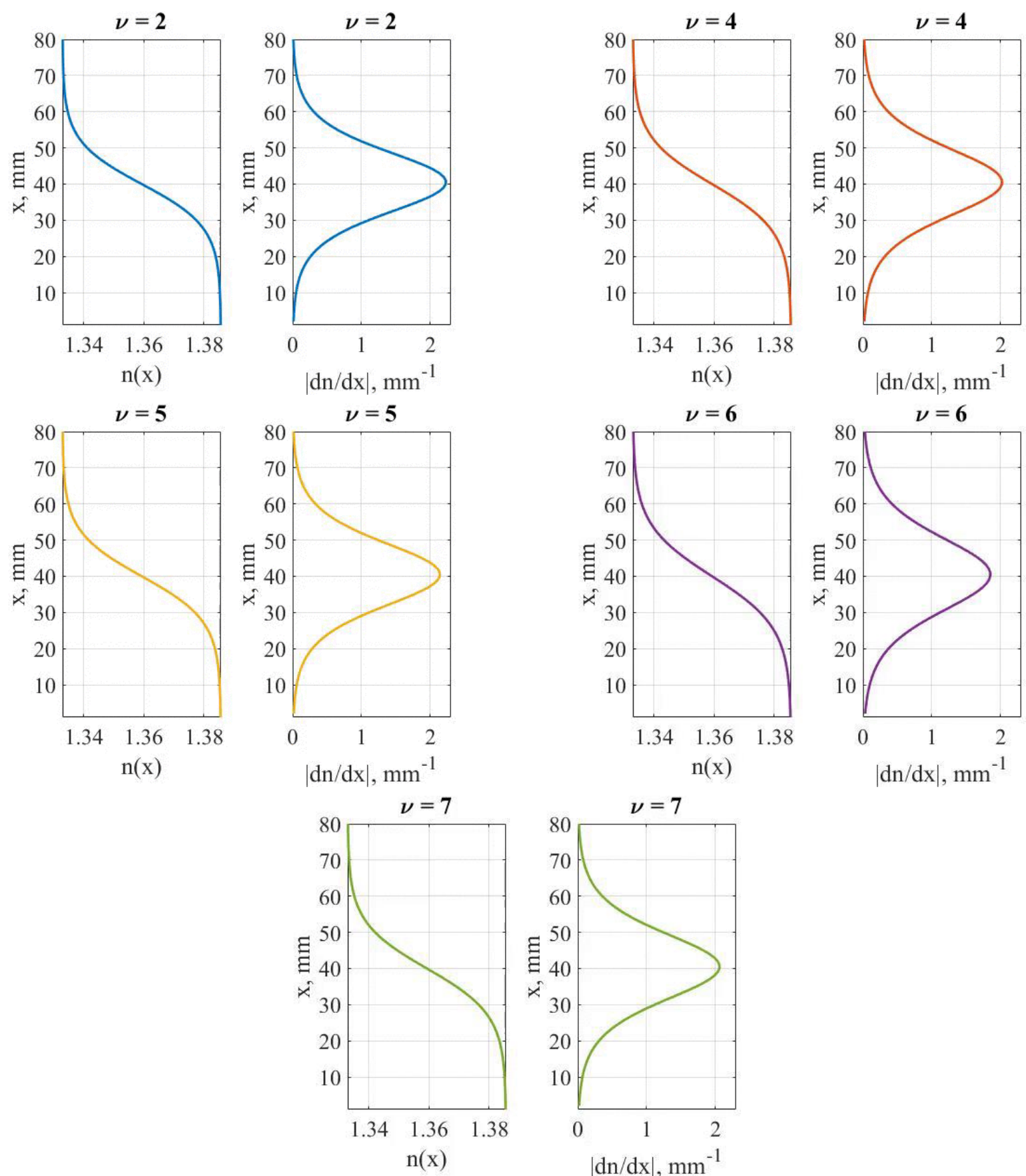

Fig. 4. Distribution change of the refractive index and the gradient of the refractive index in the diffusion layer of liquid in time for different values of the electrolyte $\mathrm{KOH}$ concentration

\section{Non-contact determination of body temperature by the position of the caustic singular points}

When convective processes at the surface of heated or cooled bodies in a liquid are studied, the correlation processing of the refractive images of the structured beams makes it possible to restore the temperature distribution in the boundary layer. However, in a number of practical applications, only the knowledge of the actual surface temperature of the body is required. In this case, to conduct contactless control of the thermophysical characteristics of the process, it is advisable to use the empirical dependences of the surface temperature on the position of the singular points of the observed caustics [3]. To determine the position of the caustic singular points, structured laser radiation is used. 
In [11], it was shown that the type of a refractogram depends on the temperature difference between the ball and water. When a boundary layer is probed near a cooled ball by a horizontal element of a structured beam, at a distance where the "beak" of the caustic is located, a special point appears on the refractogram (Fig. 5). Figure 6 shows the theoretical dependences of the position of the caustic singular point on the surface temperature of the ball for various values of water temperature.

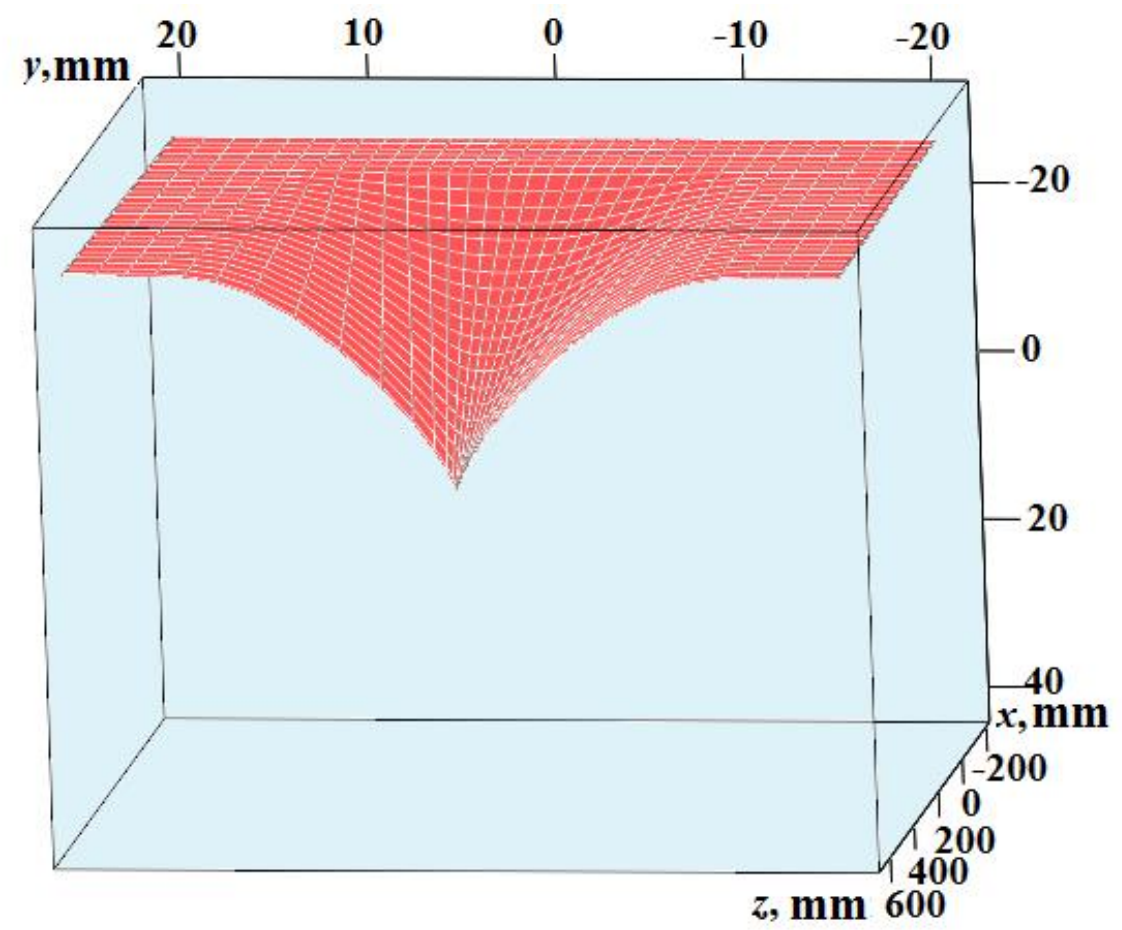

Fig. 5. Type of refractogram describing the position of the caustic's "beak"

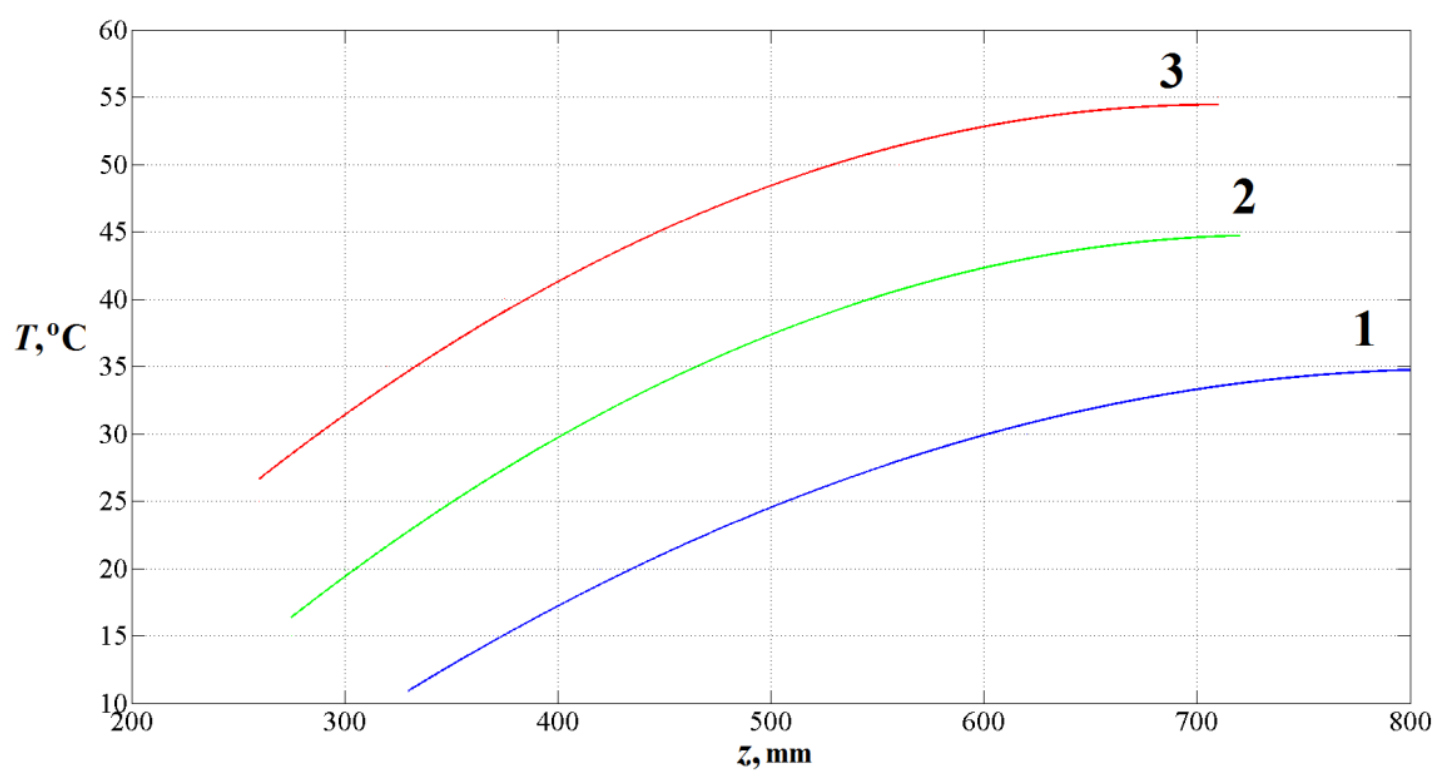

Fig. 6. Theoretical dependences of the position of the caustic singular point on the temperature of the body surface at different values of water temperature

$$
1-T_{0}=50^{\circ} \mathrm{C}, 2-T_{0}=60^{\circ} \mathrm{C}, 3-T_{0}=90^{\circ} \mathrm{C}
$$

To register caustics and to obtain the dependence of the position of the "beak" on the surface temperature, an experimental setup was created, the scheme of which is shown in Fig. 7. 


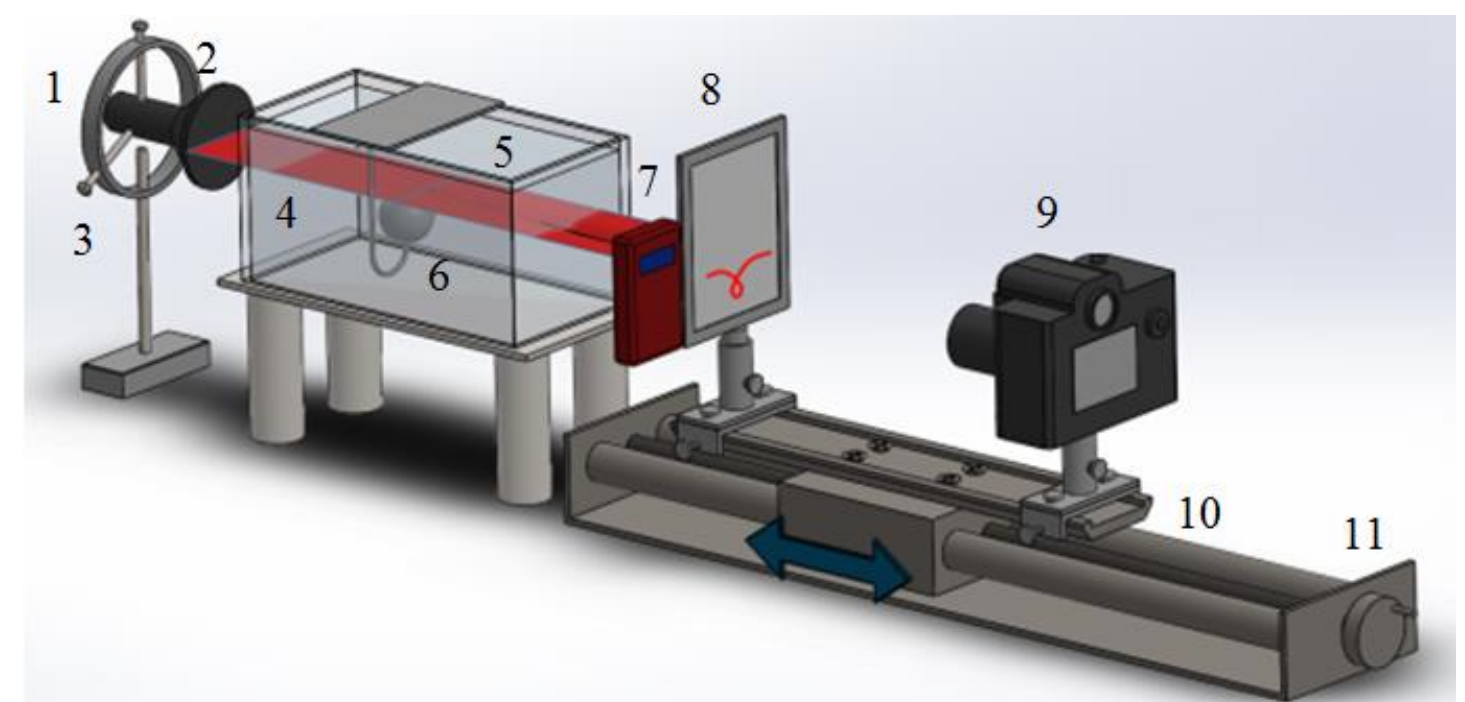

Fig. 7. Diagram of the experimental setup for obtaining the dependence of the position of the caustic's "beak" on the object surface temperature

1 - laser, 2 - optical system for the formation of a plane laser beam;

3 - tripod; 4 - laser plane, 5 - transparent cuvette with water,

6 -metal ball with a thermocouple sealed in, 7 -laser range finder,

8 - screen, 9 - digital video camera, 10 - optical rails, 11 - linear translator

A semiconductor laser 1 with a wavelength of $\lambda=650 \mathrm{~nm}$ was used as a radiation source. At the output of the laser, an optical system 2 is installed that forms the laser plane 4 . Tripod 3 provides precise two-coordinate positioning of the laser plane relative to the surface of the ball 6 located in the transparent water cell 5 . The laser beam should go in a close proximity to the ball where the laser plane was focused. A laser range finder with a corner reflector measuring the distance from the screen to the center of the ball 8 was attached to the screen 8 . The caustics were recorded with a digital video camera 9. In addition to the refractograms, the laser range finder was recorded on the video. The video camera and the screen were mounted on the optical rail 10, the distance between them remained constant throughout the experiment. The optical rail was smoothly moved using a linear translator 11. The rotational speed of the screw was chosen so that at each moment of time the beak of the caustics was displayed on the screen.

Fig. 8 shows typical types of experimental refractograms.

a)

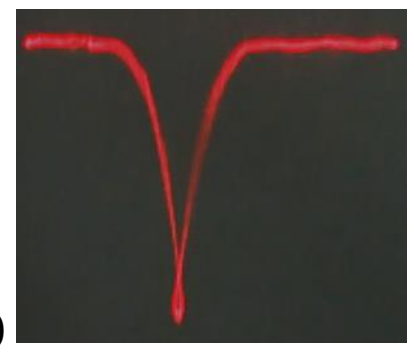

b)

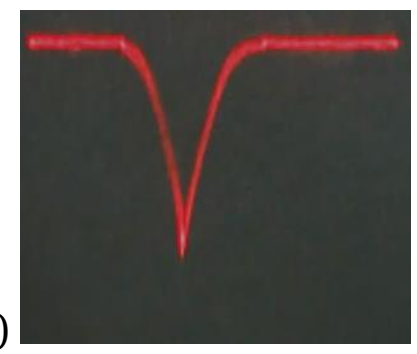

c)

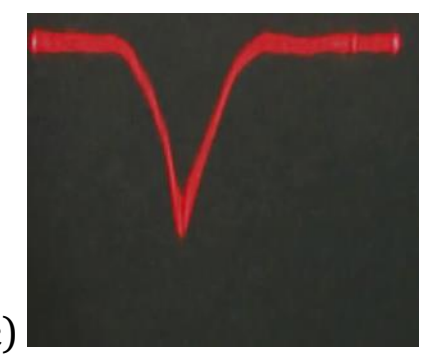

Fig. 8. Typical types of experimental refractograms of the laser plane over a cooled ball: a) loop, b) caustic "beak", c) lack of caustics

\section{Conclusion}

In this work, it was shown that the information on the localization of caustics and their geometric parameters provides additional opportunities for solving the inverse problem of restoring the properties of the medium under study. The comparison of experimentally and theoretically calculated caustic surfaces makes it possible to choose the parameters of the model describing the distribution of the refractive index in the diffusion layer of liquid. Thus, 
it can be concluded that the developed method for diagnosing the diffusion layer of a liquid allows not only to visualize the nature of the optical heterogeneity but also to determine its quantitative characteristics.

The usage of the caustic method in laser refractography to diagnose heterogeneity in liquids has shown that it is possible to determine the parameters of heterogeneous medium in a noncontact manner.

A new method to determine the surface temperature of a cold body placed in a transparent liquid is presented. The method is based on the determination of the location of singular points of caustic surfaces arising when the boundary layer is probed by a horizontal element of the structured beam. The position of the singular point of the caustic depends on the temperature difference between the surface of the body and the liquid. An additional advantage of this approach to determine the surface temperature during practical measurements is the absence of the need to specify a parametric model of the medium. The results of the work can be used in the experimental study and monitoring of inhomogeneous condensed media, when it is required to ensure non-perturbing control of the fields of concentration, pressure and temperature.

\section{Acknowledgments}

This work was supported by a grant from the President of the Russian Federation for the state support of young Russian scientists MK-6361.2018.8.

\section{References}

1. Evtikhieva O.A., Raskovskaya I.L., Rinkevichyus B.S. Laser refractography. M .: FIZMATLIT, 2008. $176 \mathrm{p}$.

2. Settles G.S. Schlieren and Shadowgraph Techniques, Visualizing Phenomena in Transparent Media, Springer, New York, 2001, P. 387

3. Raskovskaya I.L. Refractometry of optically inhomogeneous media based on recording the position of caustics by using structured laser radiation. Avtometriya, 2014, 50 (5), P. 92-98.

4. Raskovskaya I.L. Specific Imaging of Caustics upon Refraction of Structured Laser Radiation in Stratified Media // Technical Physics, 2015, 60(6), P. 911-916.

5. Diffraction Computer Optics / Ed. V.A. Soyfer. M .: Fizmatlit, 2007. 736 p.

6. Vedyashkina A.V., Rinkevichyus B.S. 3D-visualization of Caustics' Formation in Laser Refractography Problems // Physics Procedia, 2015, Vol. 73, P. 205-210.

7. Rinkevichyus B.S., Vedyashkina A.V., Pavlov I.N., Raskovskaya I.L., Tolkachev A.V. Visualization of dynamic thermal processes in transparent media using structured laser radiation // Scientific Visualization, 2016, vol. 8, no. 4, p. 50-66.

8. Raskovskaya I.L., Rinkevichyus B.S., Tolkachev A.V. Laser refraction method of visualization and quantitative diagnosis of diffusion layer of liquid in the presence of internal waves // Scientific Visualization, 2015, vol. 7, No. 4, p. 76-86.

9. Krikunov A.V., Raskovskaya I.L. B.S. Rinkevichus. Refraction of an astigmatic laser beam in a transition layer of a stratified fluid, Optics and Spectroscopy, 2011, Vol. 111, No. 6, P. 1020-1026.

10. Raskovskaya I.L., Rinkevichyus B.S., Tolkachev A.V. Laser refraction thermometry of transparent solids with inhomogeneous heating // Measurement Techniques, 2017, Vol. 59. No 10. P. 1084-1087.

11. Raskovskaya I.L., Vedyashkina A.V., Zelepukina E.V. Determination of parameters of stratified media using caustic registration with the refraction of structured laser radiation // Optical methods for studying fluxes: XIII ISTC: conference proceedings, 2015, p. 78-86.

12. Vedyashkina A.V., Pavlov I.N., Raskovskaya I.L., Rinkevichyus B.S. Experimental and computer 3D-visualization's dynamics of optical caustics in inhomogeneous mediums // Proceedings of 16 ISFV, 2014, report ISFV16-1178. 
13. Vedyashkina A.V., Rinkevichyus B.S., Raskovskaya I.L., Pavlov I.N. Laser radiation caustics method for quantitative diagnostic of transparent inhomogeneous media // Progress in Electromagnetics Research Symposium 2017, P. 754-758.

14. Gdoutos E.E. The optical method of caustics for the solution of fracture mechanics problems // 15th International Conference on Experimental Mechanics, 2012, Paper 3819.

15. Gao G., Li Z., Negahbanb M. Dynamic fracture analysis of polycarbonate by the optical method of caustics // Procedia Materials Science, 2014, No 3, P. 165-176.

16. Markides C., Kourkoulis S. Revisiting the Reflected Caustics Method: the Accurate Shape of the "Initial Curve" // Engineering transactions, 2013, 61(4, P. 265-287.

17. Vedyashkina A.V., Rinkevichyus B.S. 3D-visualization of caustics' formation in laser refractography problems // Physics Procedia "4th International Conference of Photonics and Information Optics, PhIO 2015", 2015, P. 205-210.

18. Krikunov A.V., Raskovskaya I.L., Rinkevichus B.S. Refraction of an astigmatic laser beam in the transition layer of a stratified fluid // Optics and Spectroscopy, 2011, Vol. 111, No. 6. P. 1001-1006. 